\title{
Article \\ Characterization of the Candida glabrata Transcription Factor CgMar1: Role in Azole Susceptibility
}

\author{
Pedro Pais 1,2,3 ${ }^{D}$, Mónica Galocha ${ }^{1,2,3}$, Raquel Califórnia 1,2,3 ${ }^{D}$, Romeu Viana ${ }^{1,2,3}$, Mihaela Ola ${ }^{4}$, \\ Michiyo Okamoto ${ }^{5}$, Hiroji Chibana ${ }^{5}$, Geraldine Butler ${ }^{4}(\mathbb{D}$ and Miguel C. Teixeira $1,2,3, *(\mathbb{D})$
}

1 Department of Bioengineering, Instituto Superior Técnico, Universidade de Lisboa, 1049-001 Lisboa, Portugal; pedrohpais@tecnico.ulisboa.pt (P.P.); monicagalocha@tecnico.ulisboa.pt (M.G.); raquelsc1995@hotmail.com (R.C.); romeuviana@tecnico.ulisboa.pt (R.V.)

2 iBB-Institute of Bioengineering and Biosciences, Biological Sciences Research Group, Instituto Superior Técnico, 1049-001 Lisboa, Portugal

3 Associate Laboratory i4HB-Institute for Health and Bioeconomy at Instituto Superior Técnico, Universidade de Lisboa, 1049-001 Lisboa, Portugal

4 School of Biomedical and Biomolecular Sciences, Conway Institute, University College Dublin, Dublin 4, Ireland; mihaela.ola@ucdconnect.ie (M.O.); gbutler@ucd.ie (G.B.)

5 Medical Mycology Research Center (MMRC), Chiba University, Chiba 263-8522, Japan; m-sato_okamoto@chiba-u.jp (M.O.); chibana@faculty.chiba-u.jp (H.C.)

* Correspondence: mnpct@tecnico.ulisboa.pt

\section{check for}

updates

Citation: Pais, P.; Galocha, M.;

Califórnia, R.; Viana, R.; Ola, M.;

Okamoto, M.; Chibana, H.; Butler, G.; Teixeira, M.C. Characterization of the Candida glabrata Transcription Factor CgMar1: Role in Azole Susceptibility. J. Fungi 2022, 8, 61. https://doi.org/ $10.3390 /$ jof 8010061

Academic Editors: Rajendra Prasad, Anuradha Chowdhary and

Dominique Sanglard

Received: 21 December 2021

Accepted: 5 January 2022

Published: 7 January 2022

Publisher's Note: MDPI stays neutral with regard to jurisdictional claims in published maps and institutional affiliations.

Copyright: (C) 2022 by the authors. Licensee MDPI, Basel, Switzerland. This article is an open access article distributed under the terms and conditions of the Creative Commons Attribution (CC BY) license (https:// creativecommons.org/licenses/by/ $4.0 /)$.

\begin{abstract}
The prevalence of antifungal resistance in Candida glabrata, especially against azole drugs, results in difficult-to-treat and potentially life-threatening infections. Understanding the molecular basis of azole resistance in C. glabrata is crucial to designing more suitable therapeutic strategies. In this study, the role of the transcription factor encoded by ORF CAGL0B03421g, here denominated as CgMar1 (Multiple Azole Resistance 1), in azole susceptibility was explored. Using RNA-sequencing, CgMar1 was found to regulate 337 genes under fluconazole stress, including several related to lipid biosynthesis pathways. In this context, CgMar1 and its target $C g R S B 1$, encoding a predicted sphingoid long-chain base efflux transporter, were found to contribute to plasma membrane sphingolipid incorporation and membrane permeability, decreasing fluconazole accumulation. CgMar1 was found to associate with the promoter of $C g R S B 1$, which contains two instances of the CCCCTCC consensus, found to be required for $C g R S B 1$ activation during fluconazole stress. Altogether, a regulatory pathway modulating azole susceptibility in C. glabrata is proposed, resulting from what appears to be a neofunctionalization of a Hap1-like transcription factor.
\end{abstract}

Keywords: Candida glabrata; azole resistance; transcription regulatory networks; CgMar1; transcriptomics; CgRsb1

\section{Introduction}

Candida species constitute the most common cause of fungal infections, representing the fourth leading cause of nosocomial bloodstream infections in the USA [1-3]. C. albicans is the most isolated species, but the prevalence of non-albicans infections has been steadily increasing. Candida glabrata ranks as the second or third most commonly isolated pathogenic Candida spp. depending on the geographical region [4-6], at least partially due to its ability to rapidly acquire azole resistance. One reason for the emergence of resistance in C. glabrata is related to its haploid nature, which facilitates acquisition of mutations in resistance genes.

Azole antifungals are widely used in clinical practice as both treatment and prophylaxis of fungal infections [7]. Azoles inhibit ergosterol biosynthesis by targeting the Erg11 enzyme, leading to loss of plasma membrane properties (e.g., fluidity, stability, structure, asymmetry, and function) by ergosterol depletion and incorporation of the toxic sterol dimethylcholesta-8,24(28)-dien-3 $\beta, 6 \alpha$-diol (DMCDD) in the membrane [8,9]. In C. glabrata, the transcription factors (TFs) $\mathrm{CgUpc} 2 \mathrm{a}$ and $\mathrm{CgUpc} 2 \mathrm{~b}$ are major regulators of ergosterol 
biosynthesis, while in C. albicans, this pathway is controlled by a single TF (CaUpc2) [10-12]. Azole resistance in Candida spp. is typically mediated by TFs commonly referred to as azole stress response regulators. This is the case of CgPdr1 in C. glabrata and CaTac1 in C. albicans, activators of multidrug resistance transporters (MDR) from the ATP-Binding Cassette (ABC), such as CDR1 and CDR2 [13-20]. Additionally, C. albicans also carries the TF CaMrr1, which activates the MDR1 transporter from the Major Facilitator Superfamily (MFS) [21,22].

Here, the role of the C. glabrata TF encoded by the CAGLOB03421g ORF, here designated as CgMar1 (Multiple Azole Resistance 1), in azole susceptibility is analyzed. Its role in the transcriptome-wide response to fluconazole is assessed, leading to the elucidation of its role in modulating azole susceptibility, dependent on membrane sphingolipid incorporation, membrane permeability, and intracellular drug accumulation.

\section{Materials and Methods}

\subsection{Plasmids, Strains, and Growth Media}

The plasmid pGREG576 was obtained from the Drag\&Drop collection [23]. The plasmid pV1382 [24] was obtained from Addgene (Addgene plasmid\# 111436).

All C. glabrata strains used in this study are presented in Table 1. The C. glabrata parental strain KUE100 [25] and derived single deletion mutants were batch cultured at $30{ }^{\circ} \mathrm{C}$, with orbital agitation $(250 \mathrm{rpm})$ in basal medium (BM), with the following composition (per liter): $1.7 \mathrm{~g}$ of yeast nitrogen base without amino acids or NH4+ (Difco, England, UK), $20 \mathrm{~g}$ of glucose (Merck, Darmstadt, Germany), and $2.65 \mathrm{~g}$ of (NH4)2SO4 (Merck). KUE100::URA- and the derived KUE100__cgmar1::URA- strains, harboring pGREG576derived plasmids, were grown in BM. The S. cerevisiae strain BY4741 (MATa, ura3 $\Delta 0$, leu2 $\Delta 0$, his $3 \Delta 1$, and met15 $\Delta 0$ ) was obtained from Euroscarf and grown in Yeast extract-PeptoneDextrose (YPD) medium, with the following composition (per liter): $20 \mathrm{~g}$ of glucose (Merck), $20 \mathrm{~g}$ of Peptone (Merck), and $10 \mathrm{~g}$ of Yeast extract (Merck). Besides the above-indicated ingredients, the solid media contained $20 \mathrm{~g} / \mathrm{L}$ of agar (Iberagar, Barreiro, Portugal).

Table 1. C. glabrata strains used in this study.

\begin{tabular}{|c|c|c|c|}
\hline Strain & Genotype & Parent & Reference \\
\hline KUE100 & Wild type & CBS138 & [25] \\
\hline KUE100_scgmar1 & $\Delta c g m a r 1$ & KUE100 & This study \\
\hline KUE100_scghap1 & $\Delta c g h a p$ & KUE100 & This study \\
\hline KUE100_scgrsb1 & $\Delta c g r s b 1$ & KUE100 & This study \\
\hline KUE100::URA- & $\Delta c g u r a 3$ & KUE100 & {$[26,27]$} \\
\hline KUE100_scgmar1::URA- & $\Delta c g m a r 1 \Delta c g u r a 3$ & KUE100_scgmar1 & This study \\
\hline $\begin{array}{c}\text { KUE100::URA- + } \\
\text { pGREG576_PDC1_CgMAR1 }\end{array}$ & $\Delta c g u r a 3+C g M A R 1$ & KUE100::URA- & This study \\
\hline $\begin{array}{l}\text { KUE100_Acgmar1::URA- + } \\
\text { pGREG576_PDC1_CgMAR1 }\end{array}$ & $\Delta c g m a r 1 \Delta c g u r a 3+C g M A R 1$ & KUE100__cgmar1::URA- & This study \\
\hline $\begin{array}{c}\text { KUE100::URA- + } \\
\text { pYEP354_CgRSB1prom_lacZ }\end{array}$ & $\Delta c g u r a 3+$ lacZ & KUE100::URA- & This study \\
\hline $\begin{array}{c}\text { KUE100::URA- + } \\
\text { pYEP354_mutA/B/A+B_CgRSB1prom_lacZ }\end{array}$ & $\Delta c g u r a 3+$ lacZ & KUE100::URA- & This study \\
\hline
\end{tabular}

2.2. Disruption of the C. glabrata CgHAP1, CgMAR1, and CgRSB1 Genes (ORFs CAGLOK05841g, CAGLOB03421g, and CAGLOL10142g, Respectively)

The deletion of the C. glabrata genes addressed in this study was carried out in the parental strain KUE100 using the method described by Ueno et al. [28]. Genes of interest were replaced by a DNA cassette including the CgHIS3 gene, through homologous recombination. The PCR primers used to generate the replacement cassette for each gene and the primers used for PCR confirmation of gene deletion are present in Table S1. The pHIS906 plasmid including $\mathrm{CgHIS3}$ was used as a template, and transformation was performed as described previously [25]. 


\subsection{Cloning of the C. glabrata CgMAR1 Gene (ORF CAGLOB03421g)}

The pGREG576 plasmid from the Drag\&Drop collection was used as described before to clone and express the C. glabrata ORF CAGL0B03421g [26,29-31]. pGREG576 was acquired from Euroscarf and contains a galactose inducible promoter (GAL1); the yeast selectable marker URA3; and the GFP gene, encoding a Green Fluorescent Protein (GFPS65T), which allows for monitoring of the expression and subcellular localization of the cloned fusion protein. CgMAR1 DNA was generated by PCR, using genomic DNA extracted from the sequenced CBS138 C. glabrata strain. Besides a region with homology to the first 20 and last 22 nucleotides of the CgMAR1 coding region (italic), the designed primers contain nucleotide sequences with homology to the cloning site flanking regions of the pGREG576 vector (underlined). The amplified fragments were co-transformed into the parental S. cerevisiae strain BY4741 with the pGREG576 vector, previously cut with the restriction enzyme SalI to obtain the pGREG576_CgMAR1 plasmid. Since the GAL1 promoter only allows for a very low expression of downstream genes in C. glabrata, the GAL1 promoter present in the pGREG576_CgMAR1 plasmid was replaced by the constitutive $P D C 1$ C. glabrata promoter [32], giving rise to the pGREG576_PDC1_CgMAR1 plasmid. The PDC1 promoter DNA was generated by PCR, using genomic DNA extracted from the sequenced CBS138 C. glabrata strain. Besides a region with homology to 22 nucleotides in the beginning and the last 22 nucleotides of the $1000 \mathrm{bp}$ upstream region of the PDC1 coding sequence (italic), the designed primers contain nucleotide sequences with homology to the cloning site flanking regions of the pGREG576 vector (underlined). The amplified fragment was co-transformed into the parental strain BY4741 with the pGREG576_CgMAR1 plasmid, previously cut with AscI and NotI restriction enzymes to remove the GAL1 promoter, to generate the pGREG576_PDC1_CgMAR1 plasmid. The recombinant plasmids pGREG576_CgMAR1 and pGREG576_PDCI_CgMAR1 were obtained through homologous recombination in S. cerevisiae and verified by DNA sequencing. All primers used are presented in Table S1.

\subsection{Disruption of C. glabrata CgURA3 Gene (ORF CAGL0I03080g)}

The deletion of the $C$. glabrata URA3 gene encoded by ORF CAGL0I03080g was carried out in the $\Delta$ cgmar1 mutant as described before [26] using the CRISPR-Cas9 system from Vyas et al. [24]. Briefly, a $C g U R A 3$ gRNA sequence selected from the resources made available by Vyas et al. [24] was cloned in the pV1382 plasmid, previously linearized with the restriction enzyme BsmBI (New England Biolabs, Ipswich, MA, USA). The CgURA3 gRNA was obtained by oligonucleotide annealing and the product ligated into the previously linearized pV1382 plasmid to obtain the pV1382_CgURA3 vector. The construct was verified by DNA sequencing. The plasmid was transformed into the $\Delta c g$ mar1 mutant and cells were directly plated on 5-Fluoroorotic acid (5-FOA) to select for URA- cells. Sequential passages in non-selective medium (YPD) were performed to avoid detrimental effects of further Cas9 expression, and CgURA3 loss of function was further confirmed by the inability to grow in medium without uracil. The introduction of pGREG576-derived plasmids (containing a URA3 selection marker) in the edited strains was able to rescue the growth impairment in the absence of uracil. All primers used are presented in Table S1.

\subsection{Antifungal Susceptibility Assays}

KUE100 C. glabrata and the derived deletion mutant cell suspensions used to inoculate agar plates were mid-exponential cells grown in BM, as were the KUE100::URA- and the derived deletion mutant KUE100__cgmar1::URA-. Cells were grown until a culture OD600nm of $0.5 \pm 0.05$ was reached and then diluted in sterile water to obtain suspensions with OD600nm $=0.05 \pm 0.005$. These cell suspensions and subsequent dilutions $(1: 5 ; 1: 25)$ were applied as $4 \mu \mathrm{L}$ spots onto the surface of solid BM plates supplemented with distinct chemical stress concentrations. The tested drugs included the following compounds, used in the following specified concentration ranges: the azole antifungal drugs ketoconazole (10 
to $60 \mathrm{mg} / \mathrm{L}$ ), fluconazole (100 to $250 \mathrm{mg} / \mathrm{L})$, miconazole $(0.10$ to $0.50 \mathrm{mg} / \mathrm{L})$, itraconazole (15 to $30 \mathrm{mg} / \mathrm{L})$, and clotrimazole (2.5 to $15 \mathrm{mg} / \mathrm{L})$.

MIC assays were performed in 96-well plates containing RPMI-1640 2\% glucose medium with the appropriate drug concentrations as described previously and according to EUCAST guidelines [29].

\subsection{Total RNA Extraction}

C. glabrata strains KUE100 and KUE100__cgmar1 were grown in BM until mid-exponential phase. Subsequently, the cells were transferred to fresh medium (control), or fresh medium containing $150 \mathrm{mg} / \mathrm{L}$ of fluconazole and harvested after 1h of incubation. Total RNA was isolated using an Ambion Ribopure-Yeast RNA kit according to manufacturer's instructions. The chosen concentration of fluconazole is higher than the MIC attained by microdilution testing. Resistance measurements can vary according to distinct experimental conditions, such as cell density/inoculum size, which has been demonstrated to impact inhibitory concentrations of antifungal drugs (i.e., higher cell densities result in lower drug susceptibility) [33]. The selected concentration was found to be inhibitory under the conditions used for cell growth in this study.

\subsection{Library Preparation and Gene Expression Analysis}

Strand-specific RNA-seq library preparation and sequencing was carried out as a paid service by the NGS core from Oklahoma Medical Research Foundation, Oklahoma City, Oklahoma. Libraries were generated using the Illumina Truseq Stranded Total RNA library prep kit with ribosomal depletion via RiboZero Gold according to the manufacturer's protocol. Raw data are available at GEO under accession number: GSE163158. Raw data for the wild type were obtained concurrently and has been previously submitted [29]. Bioinformatics analysis was performed as described previously [26,29]. Briefly, sample reads were trimmed using Skewer (v0.2.2) [34] and aligned to the C. glabrata CBS138 reference genome, obtained from the Candida Genome Database (CGD), using TopHat (v2.1.1) [35] with the parameters - p 12 (number of threads), - g 1 (maximum amount of times that a read can be mapped to the genome), -b2-very-sensitive (preset option), and - library-type fr-firststrand (to account for strand specificity). HTSeq (v0.7.1) [36] was used to count the mapped reads per gene. Differentially expressed genes were identified using DESeq2 [37] with an adjusted $p$-value threshold of 0.05 and a $\log 2$ fold change greater than 0.5 or less than -0.5 . Default parameters in DESeq2 were used.

\subsection{NBD-DHS Subcellular Localization Assessment}

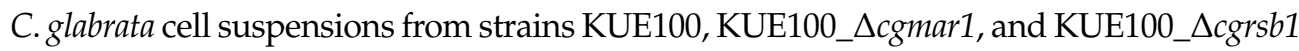
were prepared in BM until a standard culture OD600nm of $0.5 \pm 0.05$ was reached. C6 NBD-dihydrosphingosine (NBD-DHS; $1 \mathrm{mg} / \mathrm{mL}$ in methanol; Santa Cruz Biotechnology, Santa Cruz, CA, USA) was added to $1 \mathrm{~mL}$ of $4 \times 10^{7}$ cells $/ \mathrm{mL}$ to a final concentration of $5 \mu \mathrm{M}$, and cell suspensions were incubated in the dark with orbital agitation (30 min, $250 \mathrm{rpm})$. Cells exposed to C6 NBD-DHS were centrifuged (17,500× $g$ for $5 \mathrm{~min})$, washed twice, and resuspended in PBS buffer to final $10^{7}$ cells $/ \mathrm{mL}$ aliquots. NBD fluorescence was detected by fluorescence microscopy in a Zeiss Axioplan microscope (Carl Zeiss MicroImaging, Oberkochen, Germany), using excitation and emission wavelengths of 395 and $509 \mathrm{~nm}$, respectively. Fluorescence images were captured using a cooled Zeiss Axiocam 503 color (Carl Zeiss Microscopy, Oberkochen, Germany). Quantification of NBD-DHS signal localization to cell membranes was performed by accessing a minimum of 100 cells per strain.

\subsection{Plasma Membrane Permeability}

Plasma membrane permeability was assessed by the passive uptake of propidium iodide (PI; 20 mM in DMSO, Invitrogen, Waltham, MA, USA) as described previously [26,29].

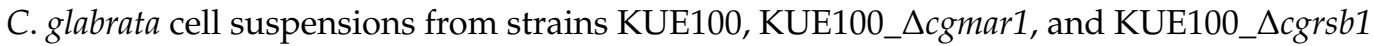


were prepared in BM until a standard culture OD600nm of $0.5 \pm 0.05$ was reached and transferred to the same medium with or without $150 \mathrm{mg} / \mathrm{L}$ of fluconazole. After $1 \mathrm{~h}$ of incubation, PI was added to $1 \mathrm{~mL}$ of $4 \times 10^{7}$ cells $/ \mathrm{mL}$ to a final concentration of $20 \mu \mathrm{M}$, and cell suspensions were incubated in the dark with orbital agitation ( $15 \mathrm{~min}, 250 \mathrm{rpm})$. Cells exposed to PI were centrifuged $(17,500 \times g$ for $5 \mathrm{~min})$, washed twice, resuspended in PBS buffer, and pooled to final $10^{7}$ cells $/ \mathrm{mL}$ aliquots. PI fluorescence was detected by fluorescence microscopy in a Zeiss Axioplan microscope (Carl Zeiss MicroImaging) using excitation and emission wavelengths of 536 and $595 \mathrm{~nm}$, respectively. Fluorescence images were captured using a cooled Zeiss Axiocam 503 color (Carl Zeiss Microscopy), and the images were analyzed with the ZEN lite software from ZEISS microscopy. Cell-to-cell fluorescence intensity was defined as the average of pixel-by-pixel intensity in the selected region of interest, and a minimum of 70 cells per strain were assessed. The fluorescence images were background corrected using dark-current images.

\subsection{0. ${ }^{3}$ H-Fluconazole Accumulation Assays}

${ }^{3} \mathrm{H}$-fluconazole transport assays were carried out as described before for other radiolabeled compounds [29-31,38]. The internal accumulation of fluconazole was determined by calculating the ratio between the radiolabeled fluconazole measured within the yeast cells and in the external medium (intracellular/extracellular). The parental strain KUE100

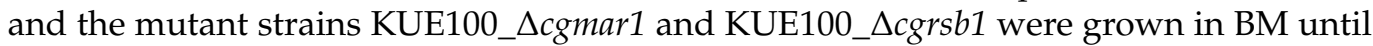
the mid-exponential phase and harvested by filtration. The cells were washed and resuspended in fresh BM to obtain dense cell suspensions (OD600 $\mathrm{nm}=0.5 \pm 0.1$, equivalent to approximately $1.57 \mathrm{mg}$ (dry weight) $\mathrm{mL}^{-1}$ ). Readily, $0.1 \mu \mathrm{M}$ of ${ }^{3} \mathrm{H}$-fluconazole (Moravek Inc., Brea, CA, USA; $1 \mathrm{mCi} / \mathrm{mL}$ ) and $150 \mathrm{mg} / \mathrm{L}$ of unlabeled fluconazole were added to the cell suspensions. Incubation proceeded for an additional period of $30 \mathrm{~min}$. The intracellular accumulation of labeled fluconazole was followed by filtering $200 \mu \mathrm{L}$ of cell suspension at adequate time intervals through pre-wetted glass microfiber filters (Whatman GF/C, Merck, Darmstadt, Germany). The filters were washed with ice-cold TM buffer, and the radioactivity was measured in a Beckman LS 5000TD scintillation counter. Extracellular ${ }^{3} \mathrm{H}$-fluconazole was estimated by radioactivity assessment of $50 \mu \mathrm{L}$ of the supernatant. Non-specific ${ }^{3} \mathrm{H}$-fluconazole adsorption to the filters and to the cells (less than $5 \%$ of the total radioactivity) was assessed and taken into consideration. To calculate the intracellular concentration of labeled fluconazole, the internal cell volume (Vi) of the exponential cells, grown in the absence of drug and used for accumulation assays, was considered constant and equal to $2.5 \mu \mathrm{L}$ (mg dry weight $)^{-1}$ [39].

\subsection{In Silico Prediction of Overrepresented Sequences in CgMar1-Activated Promoters}

The promoters ( -1000 to $-1 \mathrm{bp}$ ) upstream of the coding regions of genes for which their expressions were found to be activated by CgMar1 were retrieved using the "Retrieve Upstream Sequence" tool from PathoYeastract [40]. The obtained sequences were submitted to DREME (MEME suite) [41] for discovery of enriched sequences, using default parameters.

\subsection{Cloning of the CgRSB1 Promoter and Site-Directed Mutagenesis}

The pYPE354 plasmid was used as described before to clone and express the lacZ reporter gene [29]. pYEP354 contains the yeast selectable marker URA3 and the bacterial selectable marker AmpR genes. CgRSB1 promoter DNA was generated by PCR, using genomic DNA extracted from the sequenced CBS138 C. glabrata strain. The first primer contains a region with homology within the beginning of the $C g R S B 1$ promoter and a recognition site for the EcoRI restriction enzyme, flanked by additional bases. The second primer contains a region with homology within the end of the CgRSB1 promoter and the beginning of the $C g R S B 1$ coding sequence and a recognition site for the PstI restriction enzyme, flanked by additional bases. The amplified fragment was ligated into the pYEP354 vector (T4 Ligase, New England Biolabs), previously cut with the same restriction enzymes, 
to obtain the pYEP354_CgRSB1prom_lacZ plasmid. The putative CgMar1 consensus in the CgRSB1 promoter were mutated by site-directed mutagenesis. The designed primers contain two mutations within each potential consensus, resulting in the production of each mutated consensus by PCR amplification to obtain the pYEP354_mut_CgRSB1prom_lacZ plasmids. The original template was then degraded by DpnI digestion. All primers used are presented in Table S1.

\subsection{Gene Expression Measurement by RT-PCR}

The transcript levels of the lac $Z$ reporter gene were determined by quantitative realtime PCR (RT-PCR). KUE100::URA- cells harboring the pYEP354_CgRSB1prom_lacZ or the pYEP354_mut_CgRSB1prom_lacZ plasmids were prepared in BM until a standard culture OD600nm of $0.5 \pm 0.05$ was reached and transferred to the same medium with or without $150 \mathrm{mg} / \mathrm{L}$ of fluconazole. After $1 \mathrm{~h}$ of incubation, the cells were harvested and immediately frozen at $-80{ }^{\circ} \mathrm{C}$ until RNA extraction. For total RNA extraction, the hot phenol method was applied [42]. RT-PCR was performed as described elsewhere [26,29-31]. The synthesis of cDNA for real-time RT-PCR experiments, from total RNA samples, was performed using the MultiscribeTM reverse transcriptase kit (Applied Biosystems, Thermo Fisher Scientific, Waltham, MA, USA) and the 7500 RT-PCR Thermal Cycler Block (Applied Biosystems), following the manufacturer's instructions. The quantity of cDNA for the following reactions was kept around $10 \mathrm{ng}$. The subsequent RT-PCR step was carried out using SYBR ${ }^{\circledR}$ Green (NZYTech, Lisbon, Portugal) reagents with default parameters established by the manufacturer and the primers in Table S1. The CgRDN25 gene transcript levels were used as an internal reference.

\subsection{CgMar1 Chromatin Immunoprecipitation Assays Followed by RT-qPCR}

KUE100::URA- cells harboring the pGREG576_PDC1_CgMAR1 plasmid were grown to the mid-exponential phase in BM until $100 \mathrm{OD}$ units were reached and then transferred to a fresh medium (control) or a fresh medium containing $150 \mathrm{mg} / \mathrm{L}$ fluconazole. After $1 \mathrm{~h}$ of incubation, the cells were cross-linked with $1 \%$ formaldehyde (15 min), and cross-linking was quenched with $340 \mathrm{mM}$ glycine $(10 \mathrm{~min})$. The cells were collected, washed twice with cold PBS ( $\mathrm{pH} 7.4$ ), and frozen immediately at $-80^{\circ} \mathrm{C}$. The cell pellets were lysed with $0.5 \mathrm{~mm}$ zirconia beads (Invitrogen) in lysis buffer (50 mM HEPES (pH 7.5), $140 \mathrm{mM} \mathrm{NaCl}$, $1 \mathrm{mM}$ EDTA, 1\% Triton X-100, 0.5\% NP-40, and $0.5 \mathrm{mM}$ dithiothreitol (DTT)) by vortexing for $20 \mathrm{~s}$ and incubation on ice for $2 \mathrm{~min}$ (4 times). Cell debris was pelleted by centrifugation, and the lysate was collected. The lysates were sonicated four times for $2 \mathrm{~min}$ followed by 2 min on ice using a Bioruptor water bath sonicator. Sonicated lysates were cleared by centrifugation, and $5 \%$ of the sample was collected for use as the input (sonicated chromatin, not immunoprecipitated). For immunoprecipitation (IP), chromatin was incubated overnight at $4{ }^{\circ} \mathrm{C}$ (with rotation) with protein G SureBeads (Bio-Rad, Hercules, CA, USA) coupled with IgG anti-GFP antibody (catalog number sc-9996; Santa Cruz Biotechnology Inc.). The beads were washed according to the manufacturer's instructions, and the samples were eluted at $65^{\circ} \mathrm{C}$ for $20 \mathrm{~min}$ in Tris-EDTA (TE) buffer with $0.5 \%$ SDS. Input and immunoprecipitated samples were incubated overnight at $65^{\circ} \mathrm{C}$ for cross-linking reversal. DNA was purified with the NZYGelPure kit (NZYTech). Quantification of a specific CgRSB1 promoter region was performed by RT-PCR using $5 \mu \mathrm{L}$ of the DNA preparation from each reaction mixture. The primers used are listed in Table S1. The immunoprecipitated samples were processed together with the input samples, and the amplification product was normalized to the total template used. Based on methods reported previously $[29,43,44]$, the ratio of the immunoprecipitated promoter to the input (IP/input) was calculated, and ratios in fluconazole samples were compared with the ratio in the control samples at the specific locus of the CgRSB1 promoter.

\subsection{Used Primers}

All primers used are presented in Table S1. 


\subsection{Statistical Analysis}

All results represent the average of at least three independent experiments. Statistical analyses were performed using ANOVA with Tukey's correction (comparisons of more than two groups) or t-tests with Welch's correction (comparisons of two groups). Statistical analysis of RNA-seq data was performed using a Benjamini-Hochberg procedure. Significance levels are indicated in the figure legends $\left({ }^{*}, p<0.05 ;{ }^{* *}, p<0.01 ;{ }^{* * *}, p<0.001\right.$; and $* * * *, p<0.0001)$.

\section{Results}

\subsection{CgMar1 Affects Azole Susceptibility in Candida glabrata}

In C. albicans, the TFs CaTac1 and CaMrr1 are the main azole resistance regulators, while in C. glabrata, only CgPdr1 is recognized as taking this role. We searched for a possible CaMrr1 counterpart in C. glabrata by first assessing protein sequence similarity: the protein encoded by the uncharacterized C. glabrata ORF CAGL0B03421g shows the highest similarity hit for $C$. albicans Mrr1, although with limited identity (E-value $=10^{-20}$; $25.55 \%$ identity). Reciprocal blast attains CaMrr1 as the best hit homolog for the protein encoded by ORF CAGLOB03421 $\left(\mathrm{E}\right.$-value $=10^{-18} ; 25.55 \%$ identity). Interestingly, the ORF CAGL0B03421g has a paralog designated CgHAP1 (ORF CAGLOK05841g) and the two proteins share $38.5 \%$ identity. Syntenic context $[45,46]$ reveals that both CAGL0B03421g and CAGLOK05841g are paralogs of S. cerevisiae HAP1, resulting from genome duplication and subsequent loss of one in S. cerevisiae. Syntenic analysis also revealed the HAP1 homolog in C. albicans to be TF CaZCF20 (and not CaMRR1) despite reduced sequence similarity (Figure S1A). On the other hand, C. glabrata CAGL0B03421g/CgHap1 and C. albicans Mrr1 share some degree of sequence similarity on the DNA binding domain and a regulatory middle homology domain (Figure S1B).

Therefore, the role of the TFs CgHAP1 and CAGLOB03421g in azole stress tolerance was investigated. The deletion of CAGL0B03421g increases C. glabrata susceptibility to fluconazole, clotrimazole, ketoconazole, and miconazole and more subtly to itraconazole, when compared with the wild-type, whereas no sensitization effect was observed upon CgHAP1 deletion (Figure 1A). The MIC (Minimum Inhibitory Concentration) values for fluconazole were also determined, revealing a two-fold susceptibility increase upon deletion of CAGLOB03421g, while no difference was observed for the $\Delta c g$ hap 1 strain (Table 2). Accordingly, the expression of CAGLOB03421g rescues the mutant susceptibility phenotype, and its overexpression in wild-type cells leads to a slight decrease in susceptibility (Figure 1B). The results indicate a role for CAGL0B03421g (but not CgHAP1—CAGLOK05841g) in azole susceptibility phenotype. Since CAGLOB03421g is the syntenic HAP1 ortholog but the designation of C. glabrata Hap1 is already attributed to CAGL0K05841g (the second HAP1 homolog) and considering that $\mathrm{ScHap} 1$ is not known to participate in azole response, the gene CAGLOB03421g is henceforth designated CgMAR1 (Multiple Azole Resistance 1).

Table 2. Fluconazole susceptibility profiles determined by MIC values in deletion mutants for $C g M A R 1$ and $C g H A P 1$ and the corresponding parental strain (WT).

\begin{tabular}{cc}
\hline Strain & Fluconazole MIC (mg/L) \\
\hline WT & 16 \\
$\Delta c$ gmar1 & 8 \\
$\Delta$ cghap1 & 16 \\
\hline
\end{tabular}




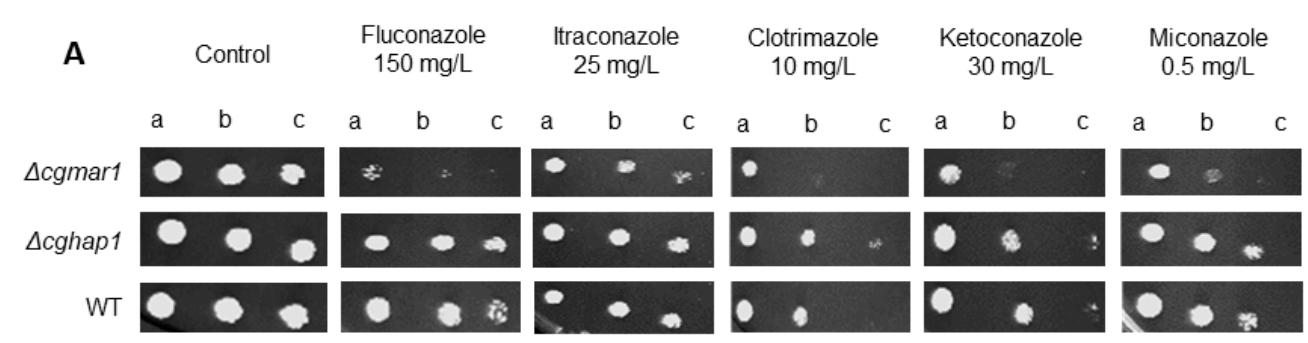

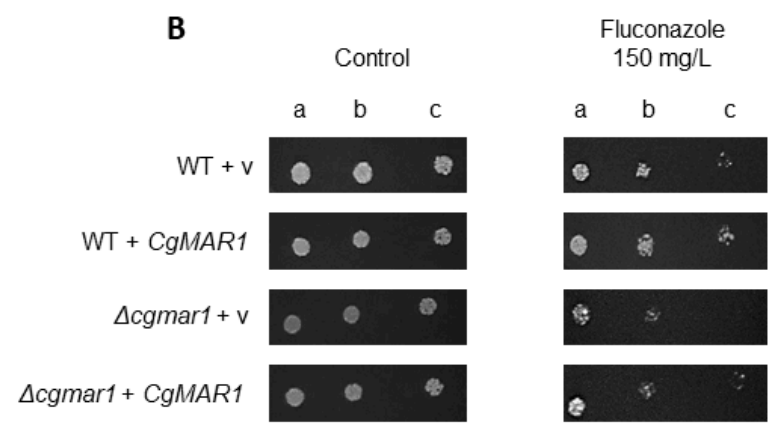

Figure 1. CgMAR1 (ORF CAGLOB03421g) mediates tolerance to fluconazole and other azoles. (A) Comparison of spot growth assays of the KUE100 C. glabrata wild-type and derived KUE100_Acghap1 and KUE100__cgmar1 deletion mutants in the presence of azole antifungals. (B) Comparison of spot growth assays of the KUE100::URA- C. glabrata wild-type and derived KUE100_Acgmar1::URA- deletion mutant harboring the pGREG576 cloning vector or the pGREG576_PDC1_CgMAR1 expression plasmid in the presence of fluconazole. The inocula were prepared as described in the Materials and Methods section. The cell suspensions used to prepare the spots were 1:5 (b) and 1:25 (c) dilutions of the cell suspensions used in (a). The images displayed are representative of at least three independent experiments.

\subsection{Role of CgMar1 in the Transcriptome-Wide Changes Occurring in Response to Fluconazole in C. glabrata}

To investigate the role of CgMar1 in fluconazole response, global gene expression changes in $\Delta$ cgmar1 cells were assessed through RNA-sequencing and compared with those of wild-type cells. In control conditions, the deletion of CgMAR1 results in the altered expression of 525 genes: 222 genes show decreased expression, while 303 genes show increased expression in $\Delta$ cgmar1 cells when compared with wild-type cells (Table S2). Interestingly, adhesin genes are among those with more prominent expression changes upon CgMAR1 deletion; however, no changes in biofilm formation were observed for the $\Delta$ cgmar 1 strain, when compared with the parental wild-type strain, in RPMI medium (Figure S2). Upon fluconazole exposure, the $\Delta$ cgmar 1 cells presented differential expression of 337 genes: 203 genes showed increased expression and 134 genes showed decreased expression when compared with wild-type cells (Table S3). Among those, 30\% were exclusively regulated during antifungal stress and not in control conditions. The most prevalent functional groups of genes putatively activated by CgMar1 (reduced expression in the $\Delta$ cgmar1 mutant) during fluconazole stress comprise carbon, nitrogen, and lipid metabolism (Figure 2A), while genes negatively regulated are especially enriched in nucleic acid processing and ribosome biogenesis genes (Figure 2B). Since the antifungal activity of fluconazole leads to defective plasma membrane properties [8,9], the differential regulation of lipid metabolism pathways by CgMar1 is particularly interesting. CgMar1-regulated genes do not include ergosterol biosynthesis but rather sterol transfer genes: CgLAM1, CgLAM5, and CgLAM6. Genes related to sphingolipid (CgRSB1 and CgYPC1) and phospho- 
lipid (CgOPI10 and CgNTE1) metabolism as well as the metabolism of carnitine (CgYAT2) and fatty acids (CgHBN1) are activated by CgMar1.

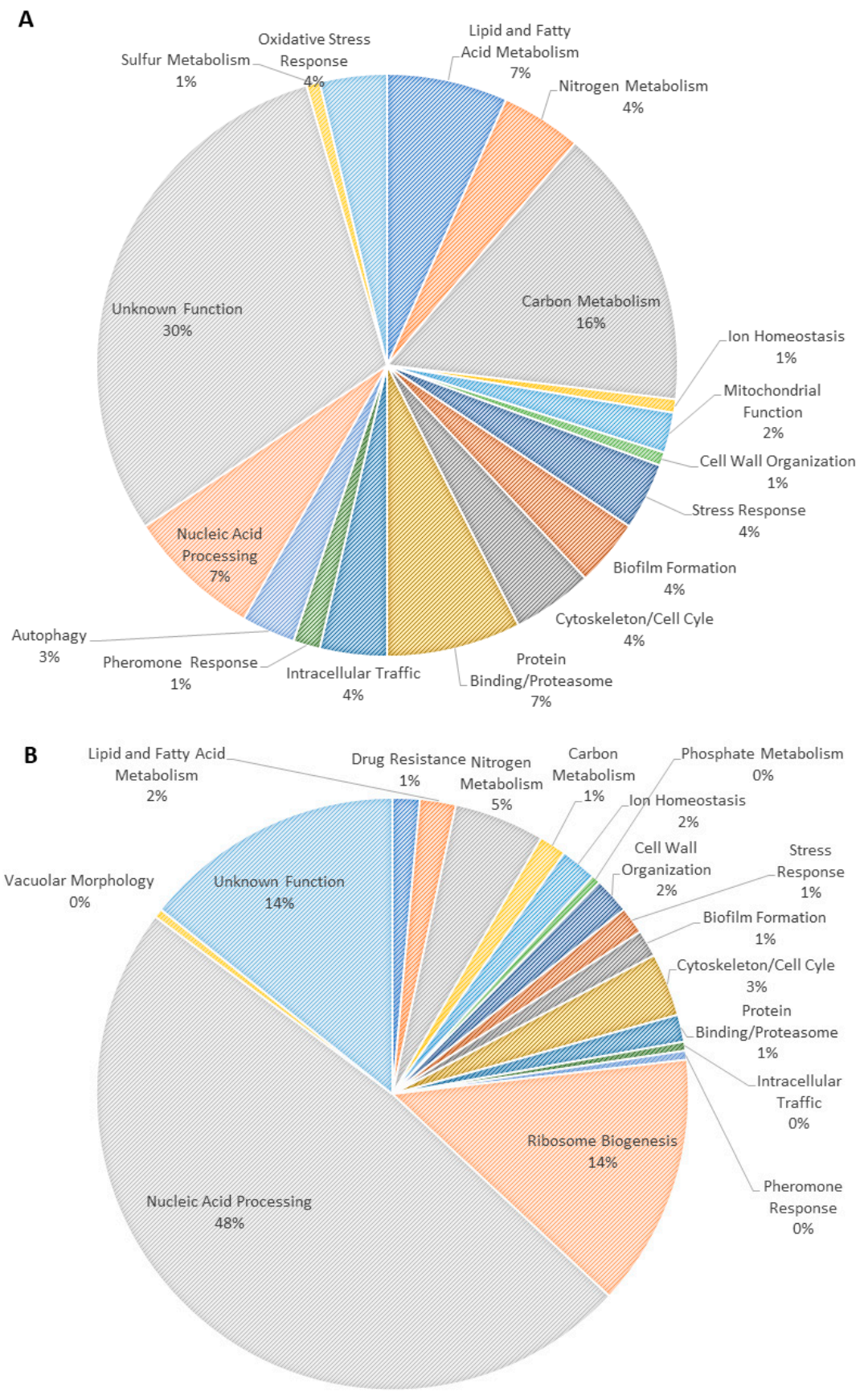

Figure 2. CgMar1-regulated functional groups. Differentially expressed genes in exponential-phase KUE100_Acgmar1 cells compared with KUE100 wild-type cells after $1 \mathrm{~h}$ of fluconazole exposure. (A) Positively CgMar1-regulated genes (downregulated in the mutant strain). (B) Negatively CgMar1regulated genes (upregulated in the mutant strain).

In the case of stress response genes, the heat-shock protein coding genes $\mathrm{CgHSP78}$ and $C g S S A 3$ are activated by CgMar1. The putative heat-shock binding protein encoded by $C g G A C 1$ is also positively regulated by CgMar1, and its C. albicans homolog was found to be induced by fluconazole [47]. Moreover, a stress responsive gene (ORF CAGL0M04763g) and a gene with a predicted function in cellular response to drug $(C g R T C 3)$ were found to be activated by CgMar1. 
Interestingly, no multidrug resistance transporters were found to be potentially activated by CgMar1. In fact, the MFS transporters CgTPO1_1 and CgTPO4, and the ABC transporter $\mathrm{CgYOR} 1$ were found to have increased expression in the $\Delta c g$ mar1 mutant. This hints that $C g M A R 1$ deletion may disrupt cellular pathways that increase drug susceptibility beyond drug transporter expression.

\subsection{CgMar1 and CgRsb1 Are Required for Sphingolipid Incorporation in the Plasma Membrane}

The previous transcriptomics analysis determined that $C g R S B 1$ expression may be, directly or indirectly, activated by CgMar1. In both S. cerevisiae and C. albicans, Rsb1 homologs (ScRsb1 and CaRta2) are members of the lipid-translocating exporter family, contributing to membrane asymmetry and sphingoid long-chain base release [48-50]. In C. glabrata, transcriptomics studies identified CgRSB1 as a CgPdr1 target [15,51-53]. Although its role in fluconazole resistance remains to be addressed, the $\Delta c g r s b 1$ mutant displays a two-fold MIC decrease in fluconazole susceptibility when compared with the wild-type (Table S4).

Therefore, we assessed the subcellular localization of fluorescently labeled dihydrosphingosine (NBD-DHS) in wild-type and $\Delta c g r s b 1$ cells. Wild-type cells incorporate DHS in the cell periphery with a dotted pattern, consistent with a lipid raft distribution in the membrane (Figure $3 \mathrm{~A}$ ). Contrarily, the $\Delta c g r s b 1$ mutant incorporates less NBD-DHS in the plasma membrane and more inside the cells (Figure 3B). The subcellular localization of NBD-DHS was also assessed in $\Delta c g m a r 1$ cells, which failed to incorporate NBD-DHS at the plasma membrane (Figure 3C). The quantification of NBD-DHS localization to cell membranes in each population supports these data: wild-type cells were seen to incorporate DHS efficiently $(85.3 \%)$, while the mutant strains $\Delta c g m a r 1$ or $\Delta c g r s b 1$ were seen to incorporate only $41.3 \%$ or $42.6 \%$ DHS in the cell membrane, respectively, which represents a two-fold decrease in membrane DHS incorporation (Figure 3D). The evidence suggests that CgRsb1 and its regulator CgMar1 contribute to the incorporation of DHS in the plasma membrane, therefore being involved in plasma membrane organization.

A

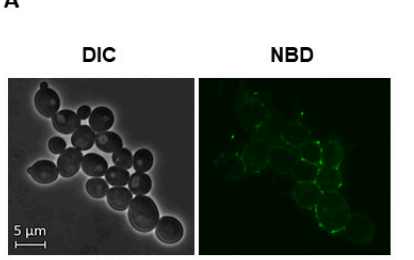

DIC

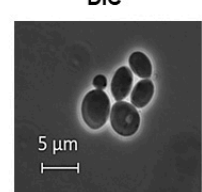

C

NBD

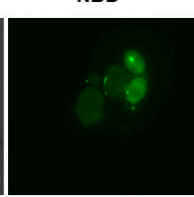

DIC

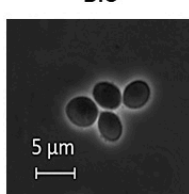

NBD

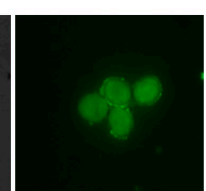

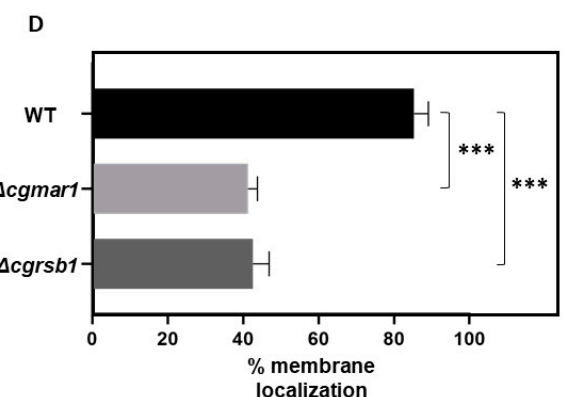

Figure 3. CgMar1 and its activated target $\mathrm{CgRsb} 1$ contribute to DHS incorporation in the plasma membrane. (A) Representative images of KUE100 C. glabrata wild-type cell fluorescence upon incorporation of NBD-DHS. (B) Representative images of KUE100_Acgrsb1 C.glabrata cell fluorescence upon incorporation of NBD-DHS. (C) Representative images of KUE100__cgmar1 C. glabrata cell fluorescence upon incorporation of NBD-DHS. (D) Percentage of cells showing membrane localization of the NBD-DHS probe. Values are the averages of at least three independent experiments. Error bars represent the corresponding standard deviations. ${ }^{* * *} p$-value $<0.001$; DIC-Differential Interference Contrast; NBD—Nitrobenzoxadiazole lipid labeling probe. 
3.4. CgMar1 and CgRsb1 Contribute to the Maintenance of Plasma Membrane Permeability and Fluconazole Accumulation of C. glabrata Cells

A possible role of CgMar1 and of its target CgRsb1 in the regulation of plasma membrane homeostasis was evaluated via measurements of cell permeability. Upon $1 \mathrm{~h}$ of fluconazole exposure, wild-type cell permeability was found to increase significantly (Figure $4 \mathrm{~A}$ ). In comparison, the permeability of $\Delta c g m a r 1$ cells was not significantly affected in control conditions; however, upon fluconazole stress, it increases to levels higher than the wild-type (Figure 4A). Accordingly, CgRSB1 deletion results in increased permeability upon fluconazole stress, comparable with the levels registered in the $\Delta$ cgmar1 (Figure 4A). This indicates that fluconazole increases C. glabrata cell permeability and that CgMar1 and CgRsb1 contribute to limiting cell permeabilization during fluconazole exposure.

A

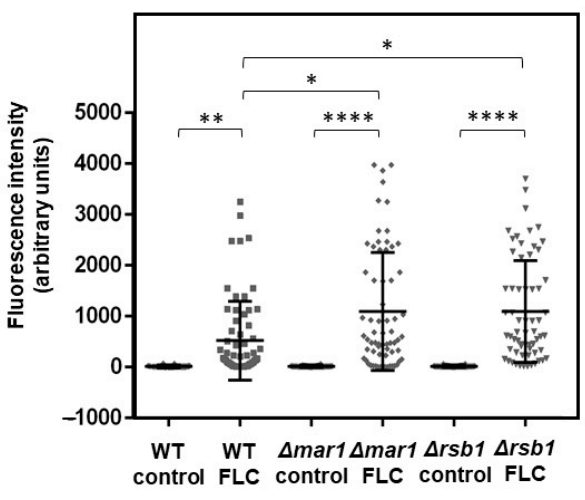

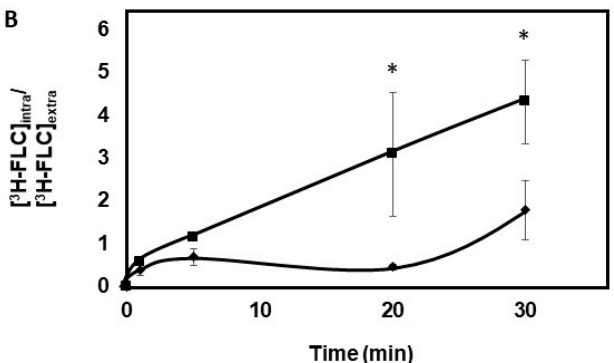

C

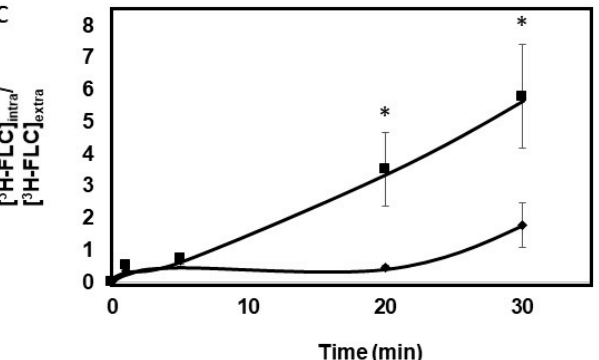

Figure 4. CgMar1 and its activated target CgRsb1 control permeability and drug accumulation in C. glabrata cells during fluconazole stress. (A) Comparison of cell permeability of the KUE100

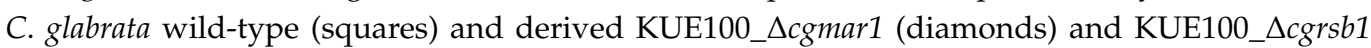
(triangles) deletion mutant cells under control conditions or after $1 \mathrm{~h}$ of fluconazole exposure. The estimation of cell permeability is based on the fluorescence intensity values exhibited by yeast cells upon the passive accumulation of propidium iodide. Error bars represent the corresponding standard deviations. (B) Time course accumulation of radiolabeled $\left[{ }^{3} \mathrm{H}\right]$ fluconazole in KUE100 C. glabrata wildtype (diamonds) and derived KUE100_Acgmar1 (squares) deletion mutant cells during cultivation in the presence of unlabeled fluconazole. (C) Time course accumulation of radiolabeled $\left[{ }^{3} \mathrm{H}\right]$ fluconazole in KUE100 C. glabrata wild-type (diamonds) and derived KUE100_Acgrsb1 (squares) deletion mutant cells during cultivation in the presence of unlabeled fluconazole. Accumulation values are the averages of at least three independent experiments. Error bars represent the corresponding standard deviations. ${ }^{*} p$-value $<0.05,{ }^{* *} p$-value $<0.01$, and ${ }^{* * *} p$-value $<0.0001$.

Consistent with the observed susceptibility and cell permeability phenotypes, the $\Delta c$ gmar1 deletion mutant accumulates 4.5 -fold more radiolabeled fluconazole than the wild-type only after 30 min of antifungal exposure (Figure 4B). These results suggest that CgMar1 activity mediates C. glabrata tolerance toward fluconazole by reducing its intracellular accumulation. The accumulation of radiolabeled fluconazole was also assessed in $\Delta c g r s b 1$ cells, found to accumulate more intracellular fluconazole than the wild-type and in levels comparable with the $\Delta c g$ mar1 mutant (Figure $4 \mathrm{C}$ ). These results indicate that CgMAR1 and CgRSB1 appear to modulate susceptibility to fluconazole by reducing drug intracellular levels. 
3.5. Identification of Nucleotide Binding Site Recognized by CgMar1 and Association with the CgRSB1 Promoter

To identify CgMar1 DNA recognition sites, the promoters of CgMar1-activated genes during fluconazole stress were searched for enriched motifs using DREME [41]. Excluding TATA-box sequences, five overrepresented consensus sequences were found (Figure S3). From those, we identified two replicates of the CCCCTCC consensus in the CgRSB1 promoter, in positions -365 to -359 and -357 to -351 .

The CgRSB1 promoter was placed upstream of the lacZ reporter gene and site-directed mutagenesis was used to disrupt each replicate of the CCCCTCC consensus (designated Motif A or Motif B, for simplicity) (Figure 5A). Upon $1 \mathrm{~h}$ of fluconazole exposure, lacZ expression increased by 1.5 -fold, which is in accordance with the transcriptomics data (Figure 5B). When either Motif A or Motif B was disrupted, lacZ expression from the mutated promoter in control conditions did not significantly change when compared with the wild-type promoter; however, under fluconazole stress, lacZ expression from the mutated promoter was reduced by two-fold compared with the wild-type promoter (Figure 5B). Additionally, when both motifs were concurrently disrupted, a more significant reduction of lacZ expression (three-fold) relative to the wild-type promoter is observed during fluconazole stress (Figure 5B). These results indicate that the two CCCCTCC motifs are required for the activation of $C g R S B 1$ expression, especially in response to fluconazole.

A

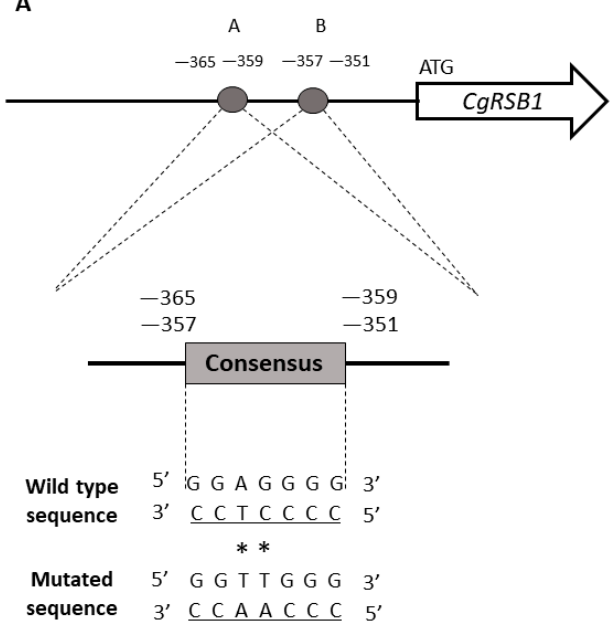

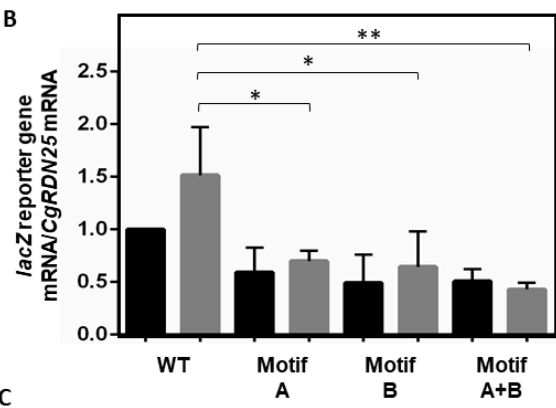

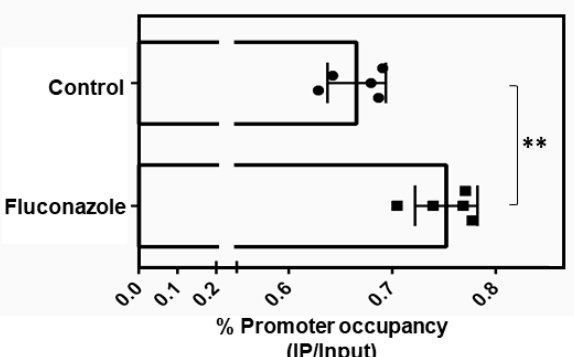

Figure 5. Possible CgMar1 DNA binding sites and association at the $C g R S B 1$ promoter. (A) The putative CgMar1 recognition sequences in the promoter of $C g R S B 1$ are in the complementary stand. The numbers refer to the position of the consensus site relative to the first ATG of the coding region. The wild-type sequence is shown underlined, with asterisks denoting the base substitutions generated by site-directed mutagenesis. The resulting mutated sequence is shown below. (B) Comparison of the variations of lacZ transcript levels determined by RT-PCR in KUE100::URA-C. glabrata cells harboring the pYEP354_CgRSB1prom_lacZ (wild-type promoter) or pYEP354_mutA/B/A+B_CgRSB1prom_lacZ (mutated promoters) plasmids under control conditions or after $1 \mathrm{~h}$ of fluconazole exposure. Transcript levels of $C g R D N 25$ were used for normalization. The black bars refer to control conditions, and the grey bars refer to fluconazole exposure. Expression values are the averages from at least three independent experiments. The error bars represent the corresponding standard deviations. (C) ChIP-RT-PCR measurements of CgMar1 promoter occupancy at the $C g R S B 1$ promoter region containing the possible recognition motifs. ChIP experiments were performed using mouse anti-GFP antibody and cultures of KUE100::URA- C. glabrata cells harboring 
the pGREG576_PDC1_CgMAR1 plasmid under control conditions or after $1 \mathrm{~h}$ of fluconazole exposure. The amplified amount of DNA was measured by RT-PCR and normalized to the total amount of the sample. Samples from the CgMar1 IP were compared to the input (IP/Input) under each condition. To determine percent occupancy, values were calculated as the ratio of the percent precipitated under fluconazole stress to the percent precipitated under control conditions. Promoter occupancy values are the averages from at least three independent experiments. Error bars represent the corresponding standard deviations. ${ }^{*} p$-value $<0.05,{ }^{* *} p$-value $<0.01$.

Subsequently, chromatin immunoprecipitation (ChIP) followed by RT-PCR was used to determine a possible association of CgMar1 to the section of the CgRSB1 promoter containing these motifs. Indeed, CgMar1 can be detected in association with the promoter of $C g R S B 1$ in the region containing the two CCCCTCC motifs in control conditions and increases its promoter occupancy upon fluconazole stress (Figure 5C).

\section{Discussion}

In this study, the TF CgMar1 (CAGLOB03421g) is characterized as a regulator of fluconazole tolerance in C. glabrata.

Deletion of $C g M A R 1$ increases susceptibility to multiple azoles, including fluconazole. The fact that differential susceptibility patterns towards the tested azoles were registered, especially in the case of itraconazole, for which only a subtle effect was observed, is consistent with the notion that azole drugs, although sharing the same primary target, may induce various side-effects and exert different levels of membrane toxicity. Still, the obtained results correlate with a previous screening where the deletion of CgMAR1 (CAGL0B03421g) was found to increase fluconazole susceptibility [54]. On the other hand, the deletion of the CgMAR1 homolog, $C g H A P 1$, leads to no phenotypic changes in relation to most azoles while inducing a modest increase in the resistance to fluconazole and clotrimazole. It would be interesting to investigate why $\mathrm{CgHap} 1$ has this effect, which may eventually be linked to its role as a heme response regulator, with heme being a crucial prosthetic group of the azole target protein, Erg11.

RNA-seq based transcriptomics revealed that CgMar1 regulates distinct cellular processes; however, it does not activate MDR transporters. Despite being a ScHAP1 homolog, CgMar1 presents a very limited activated regulon overlap with it (Figure S4). This appears to be consistent with the dissimilar CgMar1 phenotype and its putative recognition motifs predicted in our study being distinct from the established ScHap1 binding site (two CGG triplets). Among others, CgMar1 regulates genes involved in plasma membrane lipid biosynthesis and incorporation pathways, which have been correlated with azole resistance in C. albicans. In that species, an azole-resistant isolate showed altered sterol and phosphatidylcholine:phosphatidylethanolamine ratio [55]. Furthermore, response to fluconazole comprises the upregulation of sphingolipid biosynthesis genes and an altered sphingolipid composition [56]. C. albicans azole susceptibility was also associated with defective phospholipid metabolism, increased membrane fluidity, and accumulation of fluconazole [57]. Our results support similar responses in C. glabrata, as fluconazole exposure increased plasma membrane permeability. Both CgMar1 and its activated target CgRsb1 are required to control permeability levels and intracellular fluconazole accumulation. Interestingly, CgMar1 negatively affects the expression of another gene from the same lipid exporter family, CgRTA1, under control conditions. This raises the question of whether each gene may have opposite functions, for example, the encoded proteins acting as flippase/floppase. The differential activity of the multiple genes from this family may provide clarifications on membrane homeostasis.

CgMar1 and CgRsb1 contribute to the correct incorporation of sphingolipid molecules in the membrane. In C. albicans, the CaRTA2 homolog contributes to membrane homeostasis [50] and is upregulated in clinical azole-resistant isolates and in an induced fluconazoleresistant strain [58,59]. It localizes to lipid rafts and is required for the association of proteins to such rafts, including CaErg11 [60]. Furthermore, the lipid translocase CaRTA3 is required to maintain phosphatidylcholine asymmetry in the plasma membrane [61], 
is upregulated upon ketoconazole exposure [62], and is coordinately upregulated with CaCDR1 and $C a C D R 2$ during fluconazole stress [63]. This pattern is also observed in C. glabrata: CgPdr1 upregulates not only $C g C D R 1$ but also $C g R S B 1$, associating to their promoters $[15,51-53]$. The relevance of lipid flippases in azole resistance has also been demonstrated in C. neoformans [64]. This study lifts the veil on a possible involvement of CgMar1 as an additional regulator of CgRsb1, therefore justifying the correct sphingolipid incorporation in the plasma membrane and its correlation with C. glabrata susceptibility to fluconazole. However, given that membrane homeostasis impacts multiple cellular processes, including the correct sorting and function of plasma membrane proteins, a definitive mode of action for CgMar1 or CgRsb1 cannot be established yet. It is possible that these genes impact azole susceptibility indirectly by contributing to the maintenance of membrane homeostasis and, consequently, the correct action of azole resistance determinants, such as MDR transporters.

Sphingolipids also participate in antifungal resistance through interactions with ergosterol. Matched susceptible and resistant $C$. albicans clinical isolates revealed gradual changes in mannosylinositolphosphorylceramides and ergosterol [65]. Moreover, both ergosterol and the toxic sterol DMCDD integrate into the cell membrane through interaction with sphingolipids [66]. Additionally, the lipid composition of the plasma membrane can influence the activity of MDR transporters. Imbalances between membrane sphingolipids and sterols impair the sorting and functioning of $A B C$ transporters to lipid rafts [67]. Moreover, fluconazole decreases ordered lipid domains containing integral proteins and protein content [68]. Future studies could investigate imbalanced membrane compositions and the relative contributions for increased import vs. decreased transport of drugs. The increased intracellular fluconazole accumulation observed when $C g M A R 1$ or $C g R S B 1$ are deleted in C. glabrata should not be unequivocally correlated solely with antifungal import at this time as it may reflect (partially or totally) a contribution from reduced azole export prompted by defective membrane composition or structure.

Supplementary Materials: The following supporting information can be downloaded at https:/ /www.mdpi.com/article/10.3390/jof8010061/s1, Figure S1: Dot plot depicting the alignment results for the C. glabrata protein encoded by ORF CAGL0B03421g. (A) Blastp alignment result between the proteins encoded by CAGL0B03421g (C. glabrata) and CaZCF20 (C. albicans). Conserved domains and their positions are indicated at the bottom of the dot plot. (B) Blastp alignment result between the proteins encoded by CAGL0B03421g (C. glabrata) and CaMRR1 (C. albicans). Conserved domains and their positions are indicated at the bottom of the dot plot; Figure S2: Assessment of $24 \mathrm{~h}$ biofilm formation was performed by Violet Crystal in microtiter plates of the deletion mutant for CgMAR1 and the corresponding parental strain.; Figure S3: Consensus found to be overrepresented in the promoters of CgMar1-activated genes, as found by the DREME tool. The sequences shown correspond to the motifs outputted by DREME, excluding TATA box motifs; Figure S4: Comparison of the activated regulons between ScHap1 (blue) and CgMar1 (yellow), irrespective of experimental condition. The regulon of ScHap1 was retrieved from the PathoYeastract database and the C. glabrata homologs used for the comparison; Table S1: List of primers used in this study; Table S2: Differentially expressed genes in $\Delta c g m a r 1$ control/WT control; Table S3: Differentially expressed genes in $\Delta$ cgmar1 FLC/WT FLC; Table S4: Fluconazole susceptibility profiles determined by MIC values in the deletion mutant for $C g R S B 1$ and the corresponding parental strain.

Author Contributions: P.P. conducted most of the experiments and data analysis, with contributions from M.G., R.C. and R.V. and in formal analysis and software management from M.O. (Mihaela Ola) and G.B. M.O. (Michiyo Okamoto) and H.C. constructed the TF deletion mutant strains. P.P., G.B. and M.C.T. wrote the paper. M.C.T. raised funding and conceived and supervised all the work. All authors have read and agreed to the published version of the manuscript.

Funding: This research was funded by "Fundação para a Ciência e a Tecnologia” (FCT) (Contract PTDC/BII-BIO/28216/2017 and PhD grants to PP (PD/BD/113631/2015), MG (PD/BD/143027/2018), and RV (PD/BD/150586/2020)) as well as by the Programa Operacional Regional de Lisboa 2020 (LISBOA-01-0145-FEDER-022231, the BioData.pt Research Infrastructure). This work was further financed by national funds from FCT in the scope of the project UIDB/04565/2020 and UIDP/04565/2020 
of the Research Unit Institute for Bioengineering and Biosciences (iBB), project UIDB/04469/2020 for the Centre of Biological Engineering (CEB), and the project LA/P/0140/2020 of the Associate Laboratory Institute for Health and Bioeconomy (i4HB). This study was partly supported by a Joint Usage/Research Program of the Medical Mycology Research Center, Chiba University.

Institutional Review Board Statement: Not applicable.

Informed Consent Statement: Not applicable.

Data Availability Statement: RNA-sequencing data sets were deposited at the Gene Expression Omnibus, NCBI database, with the reference number GSE163158. All other data are available from the corresponding author upon reasonable request.

Conflicts of Interest: The authors declare no conflict of interest.

\section{References}

1. Azie, N.; Neofytos, D.; Pfaller, M.; Meier-Kriesche, H.-U.; Quan, S.-P.; Horn, D. The PATH (Prospective Antifungal Therapy) Alliance®registry and invasive fungal infections: Update 2012. Diagn. Microbiol. Infect. Dis. 2012, 73, 293-300. [CrossRef]

2. Wisplinghoff, H.; Bischoff, T.; Tallent, S.M.; Seifert, H.; Wenzel, R.P.; Edmond, M.B. Nosocomial Bloodstream Infections in US Hospitals: Analysis of 24,179 Cases from a Prospective Nationwide Surveillance Study. Clin. Infect. Dis. 2004, 39, 309-317. [CrossRef]

3. Edmond, M.B.; Wallace, S.E.; McClish, D.K.; Pfaller, M.A.; Jones, R.N.; Wenzel, R.P. Nosocomial Bloodstream Infections in United States Hospitals: A Three-Year Analysis. Clin. Infect. Dis. 1999, 29, 239-244. [CrossRef] [PubMed]

4. Yapar, N. Epidemiology and risk factors for invasive candidiasis. Ther. Clin. Risk Manag. 2014, 10, 95-105. [CrossRef]

5. Guinea, J. Global trends in the distribution of Candida species causing candidemia. Clin. Microbiol. Infect. 2014, 20 (Suppl. S6), 5-10. [CrossRef]

6. Tan, B.H.; Chakrabarti, A.; Li, R.Y.; Patel, A.K.; Watcharananan, S.P.; Liu, Z.; Chindamporn, A.; Tan, A.L.; Sun, P.L.; Wu, U.I.; et al. Incidence and species distribution of candidaemia in Asia: A laboratory-based surveillance study. Clin. Microbiol. Infect. 2015, 21, 946-953. [CrossRef]

7. Petrikkos, G.; Skiada, A. Recent advances in antifungal chemotherapy. Int. J. Antimicrob. Agents 2007, 30, 108-117. [CrossRef]

8. Revie, N.M.; Iyer, K.R.; Robbins, N.; Cowen, L.E. Antifungal drug resistance: Evolution, mechanisms and impact. Curr. Opin. Microbiol. 2018, 45, 70-76. [CrossRef] [PubMed]

9. Ghannoum, M.A.; Rice, L.B. Antifungal Agents: Mode of Action, Mechanisms of Resistance, and Correlation of These Mechanisms with Bacterial Resistance. Clin. Microbiol. Rev. 1999, 12, 501-517. [CrossRef] [PubMed]

10. MacPherson, S.; Akache, B.; Weber, S.; De Deken, X.; Raymond, M.; Turcotte, B. Candida albicans Zinc Cluster Protein Upc2p Confers Resistance to Antifungal Drugs and Is an Activator of Ergosterol Biosynthetic Genes. Antimicrob. Agents Chemother. 2005, 49, 2070-2083. [CrossRef]

11. Silver, P.M.; Oliver, B.G.; White, T.C. Role of Candida albicans Transcription Factor Upc2p in Drug Resistance and Sterol Metabolism. Eukaryot. Cell 2004, 3, 1391-1397. [CrossRef]

12. Nagi, M.; Nakayama, H.; Tanabe, K.; Bard, M.; Aoyama, T.; Okano, M.; Higashi, S.; Ueno, K.; Chibana, H.; Niimi, M.; et al. Transcription factors CgUPC2A and CgUPC2B regulate ergosterol biosynthetic genes in Candida glabrata. Genes Cells 2010, 16, 80-89. [CrossRef] [PubMed]

13. Vermitsky, J.-P.; Edlind, T.D. Azole Resistance in Candida glabrata: Coordinate Upregulation of Multidrug Transporters and Evidence for a Pdr1-Like Transcription Factor. Antimicrob. Agents Chemother. 2004, 48, 3773-3781. [CrossRef] [PubMed]

14. Costa, C.; Pires, C.; Cabrito, T.R.; Renaudin, A.; Ohno, M.; Chibana, H.; Sa-Correia, I.; Teixeira, M.C. Candida glabrata Drug:H+Antiporter CgQdr2 Confers Imidazole Drug Resistance, Being Activated by Transcription Factor CgPdr1. Antimicrob. Agents Chemother. 2013, 57, 3159-3167. [CrossRef]

15. Vermitsky, J.-P.; Earhart, K.D.; Smith, W.L.; Homayouni, R.; Edlind, T.D.; Rogers, P.D. Pdr1 regulates multidrug resistance in Candida glabrata: Gene disruption and genome-wide expression studies. Mol. Microbiol. 2006, 61, 704-722. [CrossRef]

16. Caudle, K.E.; Barker, K.S.; Wiederhold, N.P.; Xu, L.; Homayouni, R.; Rogers, P.D. Genomewide Expression Profile Analysis of the Candida glabrata Pdr1 Regulon. Eukaryot. Cell 2011, 10, 373-383. [CrossRef]

17. Tsai, H.-F.; Krol, A.A.; Sarti, K.E.; Bennett, J.E. Candida glabrata PDR1, a Transcriptional Regulator of a Pleiotropic Drug Resistance Network, Mediates Azole Resistance in Clinical Isolates and Petite Mutants. Antimicrob. Agents Chemother. 2006, 50, $1384-1392$. [CrossRef] [PubMed]

18. Coste, A.; Turner, V.; Ischer, F.; Morschhäuser, J.; Forche, A.; Selmecki, A.; Berman, J.; Bille, J.; Sanglard, D. A Mutation in Tac1p, a Transcription Factor Regulating CDR1 and CDR2, Is Coupled With Loss of Heterozygosity at Chromosome 5 to Mediate Antifungal Resistance in Candida albicans. Genetics 2006, 172, 2139-2156. [CrossRef]

19. Coste, A.; Selmecki, A.; Forche, A.; Diogo, D.; Bougnoux, M.-E.; D’Enfert, C.; Berman, J.; Sanglard, D. Genotypic Evolution of Azole Resistance Mechanisms in Sequential Candida albicans Isolates. Eukaryot. Cell 2007, 6, 1889-1904. [CrossRef] 
20. Coste, A.T.; Karababa, M.; Ischer, F.; Bille, J.; Sanglard, D. TAC1, Transcriptional Activator of CDR Genes, Is a New Transcription Factor Involved in the Regulation of Candida albicans ABC Transporters CDR1 and CDR2. Eukaryot. Cell 2004, 3, 1639-1652. [CrossRef]

21. Morschhäuser, J.; Barker, K.S.; Liu, T.T.; Blaß-Warmuth, J.; Homayouni, R.; Rogers, P.D. The Transcription Factor Mrr1p Controls Expression of the MDR1 Efflux Pump and Mediates Multidrug Resistance in Candida albicans. PLoS Pathog. 2007, 3, e164. [CrossRef]

22. Dunkel, N.; Blaß, J.; Rogers, P.D.; Morschhäuser, J. Mutations in the Multi-Drug Resistance Regulator MRR1, Followed by Loss of Heterozygosity, Are the Main Cause of MDR1 Overexpression in Fluconazole-Resistant Candida albicans Strains. Mol. Microbiol. 2008, 69, 827-840. [CrossRef]

23. Jansen, G.; Wu, C.; Schade, B.; Thomas, D.Y.; Whiteway, M. Drag\&Drop Cloning in Yeast. Gene 2005, 344, 43-51. [CrossRef]

24. Vyas, V.K.; Bushkin, G.G.; Bernstein, D.A.; Getz, M.A.; Sewastianik, M.; Barrasa, M.I.; Bartel, D.P.; Fink, G.R. New CRISPR Mutagenesis Strategies Reveal Variation in Repair Mechanisms among Fungi. mSphere 2018, 3, e00154-18. [CrossRef]

25. Ueno, K.; Uno, J.; Nakayama, H.; Sasamoto, K.; Mikami, Y.; Chibana, H. Development of a Highly Efficient Gene Targeting System Induced by Transient Repression of YKU80 Expression in Candida glabrata. Eukaryot. Cell 2007, 6, 1239-1247. [CrossRef]

26. Pais, P.; Vagueiro, S.; Mil-Homens, D.; Pimenta, A.I.; Viana, R.; Okamoto, M.; Chibana, H.; Fialho, A.M.; Teixeira, M.C. A New Regulator in the Crossroads of Oxidative Stress Resistance and Virulence in Candida glabrata: The Transcription Factor CgTog1. Virulence 2020, 11, 1522-1538. [CrossRef] [PubMed]

27. Cavalheiro, M.; Romão, D.; Santos, R.; Mil-Homens, D.; Pais, P.; Costa, C.; Galocha, M.; Pereira, D.; Takahashi-Nakaguchi, A.; Chibana, H.; et al. Role of CgTpo4 in Polyamine and Antimicrobial Peptide Resistance: Determining Virulence in Candida glabrata. Int. J. Mol. Sci. 2021, 22, 1376. [CrossRef] [PubMed]

28. Ueno, K.; Matsumoto, Y.; Uno, J.; Sasamoto, K.; Sekimizu, K.; Kinjo, Y.; Chibana, H. Intestinal Resident Yeast Candida glabrata Requires Cyb2p-Mediated Lactate Assimilation to Adapt in Mouse Intestine. PLoS ONE 2011, 6, e24759. [CrossRef]

29. Pais, P.; Califórnia, R.; Galocha, M.; Viana, R.; Ola, M.; Cavalheiro, M.; Takahashi-Nakaguchi, A.; Chibana, H.; Butler, G.; Teixeira, M.C. Candida Glabrata Transcription Factor Rpn4 Mediates Fluconazole Resistance through Regulation of Ergosterol Biosynthesis and Plasma Membrane Permeability. Antimicrob. Agents Chemother. 2020, 64, e00554-20. [CrossRef]

30. Pais, P.; Pires, C.; Costa, C.; Okamoto, M.; Chibana, H.; Teixeira, M.C. Membrane Proteomics Analysis of the Candida Glabrata Response to 5-Flucytosine: Unveiling the Role and Regulation of the Drug Efflux Transporters CgFlr1 and CgFlr2. Front. Microbiol. 2016, 7, 2045. [CrossRef]

31. Pais, P.; Costa, C.; Pires, C.; Shimizu, K.; Chibana, H.; Teixeira, M.C. Membrane Proteome-Wide Response to the Antifungal Drug Clotrimazole in Candida glabrata: Role of the Transcription Factor CgPdr1 and the Drug:H+ Antiporters CgTpo1_1 and CgTpo1_2. Mol. Cell. Proteom. 2016, 15, 57-72. [CrossRef]

32. Zordan, R.E.; Ren, Y.; Pan, S.-J.; Rotondo, G.; Peñas, A.D., las; Iluore, J.; Cormack, B.P. Expression Plasmids for Use in Candida glabrata. G3 Genes I Genomes I Genet. 2013, 3, 1675-1686. [CrossRef]

33. Perumal, P.; Mekala, S.; Chaffin, W.L. Role for Cell Density in Antifungal Drug Resistance in Candida albicans Biofilms. Antimicrob. Agents Chemother. 2007, 51, 2454-2463. [CrossRef]

34. Jiang, H.; Lei, R.; Ding, S.-W.; Zhu, S. Skewer: A Fast and Accurate Adapter Trimmer for next-Generation Sequencing Paired-End Reads. BMC Bioinform. 2014, 15, 182. [CrossRef]

35. Trapnell, C.; Pachter, L.; Salzberg, S.L. TopHat: Discovering Splice Junctions with RNA-Seq. Bioinformatics 2009, $25,1105-1111$. [CrossRef]

36. Anders, S.; Huber, W. Differential Expression Analysis for Sequence Count Data. Genome Biol. 2010, 11, R106. [CrossRef] [PubMed]

37. Love, M.I.; Huber, W.; Anders, S. Moderated Estimation of Fold Change and Dispersion for RNA-Seq Data with DESeq2. Genome Biol. 2014, 15, 550. [CrossRef]

38. Romão, D.; Cavalheiro, M.; Mil-Homens, D.; Santos, R.; Pais, P.; Costa, C.; Takahashi-Nakaguchi, A.; Fialho, A.; Chibana, H.; Teixeira, M.C. A New Determinant of Candida glabrata Virulence: The Acetate Exporter CgDtr1. Front. Cell. Infect. Microbiol. 2017, 7, 473. [CrossRef] [PubMed]

39. Rosa, M.F.; Sá>-Correia, I. Intracellular Acidification Does Not Account for Inhibition of Saccharomyces Cerevisiae Growth in the Presence of Ethanol. FEMS Microbiol. Lett. 1996, 135, 271-274. [CrossRef]

40. Monteiro, P.T.; Pais, P.; Costa, C.; Manna, S.; Sa-Correia, I.; Teixeira, M.C. The PathoYeastract Database: An Information System for the Analysis of Gene and Genomic Transcription Regulation in Pathogenic Yeasts. Nucleic Acids Res. 2017, 45, D597-D603. [CrossRef] [PubMed]

41. Bailey, T.L. DREME: Motif Discovery in Transcription Factor ChIP-Seq Data. Bioinformatics 2011, 27, 1653-1659. [CrossRef] [PubMed]

42. Köhrer, K.; Domdey, H. Preparation of High Molecular Weight RNA. Methods Enzymol. 1991, 194, 398-405. [PubMed]

43. Merhej, J.; Delaveau, T.; Guitard, J.; Palancade, B.; Hennequin, C.; Garcia, M.; Lelandais, G.; Devaux, F. Yap7 Is a Transcriptional Repressor of Nitric Oxide Oxidase in Yeasts, Which Arose from Neofunctionalization after Whole Genome Duplication. Mol. Microbiol. 2015, 96, 951-972. [CrossRef]

44. Knutson, B.A.; Hahn, S. Domains of Tra1 Important for Activator Recruitment and Transcription Coactivator Functions of SAGA and NuA4 Complexes. Mol. Cell. Biol. 2011, 31, 818-831. [CrossRef] 
45. Proux-Wéra, E.; Armisén, D.; Byrne, K.P.; Wolfe, K.H. A Pipeline for Automated Annotation of Yeast Genome Sequences by a Conserved-Synteny Approach. BMC Bioinform. 2012, 13, 237. [CrossRef] [PubMed]

46. Maguire, S.L.; ÓhÉigeartaigh, S.S.; Byrne, K.P.; Schröder, M.S.; O'Gaora, P.; Wolfe, K.H.; Butler, G. Comparative Genome Analysis and Gene Finding in Candida Species Using CGOB. Mol. Biol. Evol. 2013, 30, 1281-1291. [CrossRef]

47. Copping, V.M.S.; Barelle, C.J.; Hube, B.; Gow, N.A.R.; Brown, A.J.P.; Odds, F.C. Exposure of Candida Albicans to Antifungal Agents Affects Expression of SAP2 and SAP9 Secreted Proteinase Genes. J. Antimicrob. Chemother. 2005, 55, 645-654. [CrossRef]

48. Kihara, A.; Igarashi, Y. Identification and Characterization of a Saccharomyces cerevisiae Gene, RSB1, Involved in Sphingoid Long-Chain Base Release. J. Biol. Chem. 2002, 277, 30048-30054. [CrossRef]

49. Kihara, A.; Igarashi, Y. Cross Talk between Sphingolipids and Glycerophospholipids in the Establishment of Plasma Membrane Asymmetry. Mol. Biol. Cell 2004, 15, 4949-4959. [CrossRef]

50. Jia, X.M.; Wang, Y.; Jia, Y.; Gao, P.H.; Xu, Y.G.; Wang, L.; Cao, Y.Y.; Cao, Y.B.; Zhang, L.X.; Jiang, Y.Y. RTA2 Is Involved in Calcineurin-Mediated Azole Resistance and Sphingoid Long-Chain Base Release in Candida albicans. Cell. Mol. Life Sci. 2009, 66, 122-134. [CrossRef]

51. Tsai, H.-F.; Sammons, L.R.; Zhang, X.; Suffis, S.D.; Su, Q.; Myers, T.G.; Marr, K.A.; Bennett, J.E. Microarray and Molecular Analyses of the Azole Resistance Mechanism in Candida glabrata Oropharyngeal Isolates. Antimicrob. Agents Chemother. 2010, 54, 3308-3317. [CrossRef]

52. Ferrari, S.; Sanguinetti, M.; Torelli, R.; Posteraro, B.; Sanglard, D. Contribution of CgPDR1-Regulated Genes in Enhanced Virulence of Azole-Resistant Candida glabrata. PLoS ONE 2011, 6, e17589. [CrossRef] [PubMed]

53. Paul, S.; Bair, T.B.; Moye-Rowley, W.S. Identification of Genomic Binding Sites for Candida glabrata Pdr1 Transcription Factor in Wild-Type and P0 Cells. Antimicrob. Agents Chemother. 2014, 58, 6904-6912. [CrossRef]

54. Klimova, N.; Yeung, R.; Kachurina, N.; Turcotte, B. Phenotypic Analysis of a Family of Transcriptional Regulators, the Zinc Cluster Proteins, in the Human Fungal Pathogen Candida glabrata. G3 Genes I Genomes I Genet. 2014, 4, 931-940. [CrossRef]

55. Löffler, J.; Einsele, H.; Hebart, H.; Schumacher, U.; Hrastnik, C.; Daum, G. Phospholipid and Sterol Analysis of Plasma Membranes of Azole-Resistant Candida albicans Strains. FEMS Microbiol. Lett. 2000, 185, 59-63. [CrossRef]

56. Gao, J.; Wang, H.; Li, Z.; Wong, A.H.-H.; Wang, Y.-Z.; Guo, Y.; Lin, X.; Zeng, G.; Wang, Y.; Wang, J. Candida albicans Gains Azole Resistance by Altering Sphingolipid Composition. Nat. Commun. 2018, 9, 4495. [CrossRef]

57. Khandelwal, N.K.; Sarkar, P.; Gaur, N.A.; Chattopadhyay, A.; Prasad, R. Phosphatidylserine Decarboxylase Governs Plasma Membrane Fluidity and Impacts Drug Susceptibilities of Candida albicans Cells. Biochim. Biophys. Acta (BBA) Biomembr. 2018, 1860, 2308-2319. [CrossRef]

58. Barker, K.S.; Crisp, S.; Wiederhold, N.; Lewis, R.E.; Bareither, B.; Eckstein, J.; Barbuch, R.; Bard, M.; Rogers, P.D. Genome-Wide Expression Profiling Reveals Genes Associated with Amphotericin B and Fluconazole Resistance in Experimentally Induced Antifungal Resistant Isolates of Candida albicans. J. Antimicrob. Chemother. 2004, 54, 376-385. [CrossRef]

59. Jia, X.-M.; Ma, Z.-P.; Jia, Y.; Gao, P.-H.; Zhang, J.-D.; Wang, Y.; Xu, Y.-G.; Wang, L.; Cao, Y.-Y.; Cao, Y.-B.; et al. RTA2, a Novel Gene Involved in Azole Resistance in Candida albicans. Biochem. Biophys. Res. Commun. 2008, 373, 631-636. [CrossRef] [PubMed]

60. Wang, L.; Jia, Y.; Tang, R.-J.; Xu, Z.; Cao, Y.-B.; Jia, X.-M.; Jiang, Y.-Y. Proteomic Analysis of Rta2p-Dependent Raft-Association of Detergent-Resistant Membranes in Candida albicans. PLoS ONE 2012, 7, e37768. [CrossRef] [PubMed]

61. Srivastava, A.; Sircaik, S.; Husain, F.; Thomas, E.; Ror, S.; Rastogi, S.; Alim, D.; Bapat, P.; Andes, D.R.; Nobile, C.J.; et al. Distinct Roles of the 7-Transmembrane Receptor Protein Rta3 in Regulating the Asymmetric Distribution of Phosphatidylcholine across the Plasma Membrane and Biofilm Formation in Candida albicans. Cell. Microbiol. 2017, 19, e12767. [CrossRef] [PubMed]

62. Liu, T.T.; Lee, R.E.B.; Barker, K.S.; Lee, R.E.; Wei, L.; Homayouni, R.; Rogers, P.D. Genome-Wide Expression Profiling of the Response to Azole, Polyene, Echinocandin, and Pyrimidine Antifungal Agents in Candida albicans. Antimicrob. Agents Chemother. 2005, 49, 2226-2236. [CrossRef] [PubMed]

63. Whaley, S.G.; Tsao, S.; Weber, S.; Zhang, Q.; Barker, K.S.; Raymond, M.; Rogers, P.D. The RTA3 Gene, Encoding a Putative Lipid Translocase, Influences the Susceptibility of Candida albicans to Fluconazole. Antimicrob. Agents Chemother. 2016, 60, 6060-6066. [CrossRef]

64. Huang, W.; Liao, G.; Baker, G.M.; Wang, Y.; Lau, R.; Paderu, P.; Perlin, D.S.; Xue, C. Lipid Flippase Subunit Cdc50 Mediates Drug Resistance and Virulence in Cryptococcus Neoformans. mBio 2016, 7, e00478-16. [CrossRef]

65. Singh, A.; Yadav, V.; Prasad, R. Comparative Lipidomics in Clinical Isolates of Candida Albicans Reveal Crosstalk between Mitochondria, Cell Wall Integrity and Azole Resistance. PLoS ONE 2012, 7, e39812. [CrossRef] [PubMed]

66. Bittman, R.; Kasireddy, C.R.; Mattjus, P.; Slotte, J.P. Interaction of Cholesterol with Sphingomyelin in Monolayers and Vesicles. Biochemistry 1994, 33, 11776-11781. [CrossRef] [PubMed]

67. Prasad, R.; Khandelwal, N.K.; Banerjee, A. Yeast ABC Transporters in Lipid Trafficking. Fungal Genet. Biol. 2016, 93, 25-34. [CrossRef]

68. Sgherri, C.; Porta, A.; Castellano, S.; Pinzino, C.; Quartacci, M.F.; Calucci, L. Effects of Azole Treatments on the Physical Properties of Candida Albicans Plasma Membrane: A Spin Probe EPR Study. Biochim. Biophys. Acta (BBA) Biomembr. 2014, 1838, 465-473. [CrossRef] [PubMed] 